\title{
Determination of Diffusion Coefficients of Lithium in Solid Electrolyte LiPON
}

\author{
Alexander Rudy ${ }^{1, *}$, Alexander Mironenko ${ }^{1}$, Victor Naumov ${ }^{1}\left(\right.$, Alena Novozhilova ${ }^{1}$, Alexander Skundin ${ }^{2}$ \\ and Ivan Fedorov ${ }^{1}$ \\ 1 Department of Physics, P.G. Demidov Yaroslavl State University, Sovetskaya St., 14, 150003 Yaroslavl, Russia; \\ amironenko55@mail.ru (A.M.); vvnau@rambler.ru (V.N.); alena.novozhilova.2014@mail.ru (A.N.); \\ fis444@rumbler.ru (I.F.) \\ 2 Frumkin Institute of Physical Chemistry and Electrochemistry, Leninskii Ave., 31, bld. 4, \\ 119071 Moscow, Russia; office@phyche.ac.ru \\ * Correspondence: rudy@uniyar.ac.ru
}

Citation: Rudy, A.; Mironenko, A.; Naumov, V.; Novozhilova, A.;

Skundin, A.; Fedorov, I. Determination of Diffusion Coefficients of Lithium in Solid Electrolyte LiPON. Batteries 2021, 7, 21. https://doi.org/10.3390/ batteries7020021

Academic Editor: Carolina RoseroNavarro

Received: 19 November 2020

Accepted: 9 March 2021

Published: 29 March 2021

Publisher's Note: MDPI stays neutral with regard to jurisdictional claims in published maps and institutional affiliations.

Copyright: (c) 2021 by the authors. Licensee MDPI, Basel, Switzerland. This article is an open access article distributed under the terms and conditions of the Creative Commons Attribution (CC BY) license (https:// creativecommons.org/licenses/by/ $4.0 /)$.

\begin{abstract}
A structural model of LiPON solid electrolyte, containing elements that simulate drift conductivity, diffusion conductivity, and leakage current was proposed. The dependence of the impedance of the structural model on frequency was calculated, and the parameters of the model at which the theoretical curve best approximates the experimental Nyquist diagrams were determined. Based on these data, the ion diffusion coefficient and conductivity of LiPON were calculated, which are $D_{1}=1.5 \times 10^{-11} \mathrm{~cm}^{2} / \mathrm{s}$ and $\sigma=1.9 \times 10^{-6} \mathrm{~S} / \mathrm{cm}$, respectively.
\end{abstract}

Keywords: solid electrolyte; diffusion coefficient; conductivity; impedance spectroscopy; warburg element

\section{Introduction}

The expansion of the market for miniature and wearable electronic devices, such as smartphones, wrist gadgets, transdermal patches, RFID tags, and smart cards, is driving the demand for solid state Li ion battery (SSLIB). In the overwhelming majority of commercially available batteries, phosphorus-lithium oxynitride (LiPON) is used as a solid electrolyte. It should be noted that the specific capacity of SSLIB is significantly lower than the capacity of batteries with liquid electrolytes. Thus, in the SSLIB of electrochemical system $\mathrm{Li}_{\mathrm{x}} \mathrm{V}_{2} \mathrm{O}_{5} / \mathrm{LiPON} / \mathrm{Si} @ \mathrm{O} @ \mathrm{Al}$ [1], the specific capacity is $5.6 \mu \mathrm{A} \cdot \mathrm{h} / \mathrm{cm}^{2}$ and $6.5 \mathrm{~mA} \cdot \mathrm{h} / \mathrm{cm}^{3}$, while the thin-film cells of the same system with a liquid electrolyte have a maximum capacity of $200 \mu \mathrm{A} \cdot \mathrm{h} / \mathrm{cm}^{2}$ and $20 \mathrm{~mA} \cdot \mathrm{h} / \mathrm{cm}^{3}$. The decrease in capacity is due to the lower ionic conductivity of the solid LiPON electrolyte compared to the liquid electrolyte. The conductivity of a solid electrolyte depends on the concentration of free lithium ions and their mobility. In this case, the main reason for the low conductivity is the low mobility of lithium ions, which is related to the diffusion coefficient by the Einstein relation. In addition, in electrolytes, a stationary electric field is screened by charge carriers and charge transfer occurs mainly due to diffusion. It follows that the ionic conductivity of a solid electrolyte is determined by the diffusion of lithium ions; therefore, the diffusion coefficient is as important an electrolyte characteristic as its conductivity.

To determine the diffusion coefficient of lithium ions and calculate the ionic conductivity, a Pt/LiPON/Pt structure was fabricated and studied by impedance spectroscopy method. Below is a description of the $\mathrm{Pt} / \mathrm{LiPON} / \mathrm{Pt}$ manufacturing process, the results of impedance spectroscopy, and their interpretation based on the proposed structural model. By fitting the parameters of the structural model, the Warburg amplitudes for diffusion elements were determined and the diffusion coefficient of lithium ions and LiPON conductivity were calculated. 


\section{Results}

\subsection{Experimental Samples}

LiPON films (Figure 1) were deposited on the oxidized silicon wafer at a SCR-651 "Tetra" unit by magnetron sputtering of lithium orthophosphate under a nitrogen flow (18.6 standard cubic $\mathrm{cm} / \mathrm{min}$ ) at a residual pressure of $2 \mu \mathrm{bar}$. The magnetron power was $150 \mathrm{~W}$, the deposition time was $3 \mathrm{~h}$, and the thickness of the deposited film was $1 \mu \mathrm{m}$. The thickness of the platinum electrodes, deposited by magnetron sputtering, was $100 \mathrm{~nm}$. Copper foil strips, attached to platinum electrodes by electroconductive glue, served as down conductors. The nitrogen concentration in the LiPON films, according to the data of energy dispersive analysis (INCAx-act EDA), was 16.8 at.\% at an accelerating voltage of $5 \mathrm{kV}$ and 16.5 at.\% at a voltage of $7 \mathrm{kV}$ (Figure 2). According to $\mathrm{X}$-ray phase analysis (ARL X'tra), the LiPON films were amorphous (Figure 3). Figure 4 shows the cleavage of a $\operatorname{Pt}(100 \mathrm{~nm}) / \mathrm{LiPON}(1 \mu \mathrm{m}) / \mathrm{Pt}(100 \mathrm{~nm})$ cell. The inset shows a section of the solid LiPON electrolyte layer. The impedance spectrum obtained on a multichannel potentiostatgalvanostat P-20X8 in the frequency range of $1 \mathrm{~Hz}$ to $20 \mathrm{kHz}$ is shown in Figure 5.

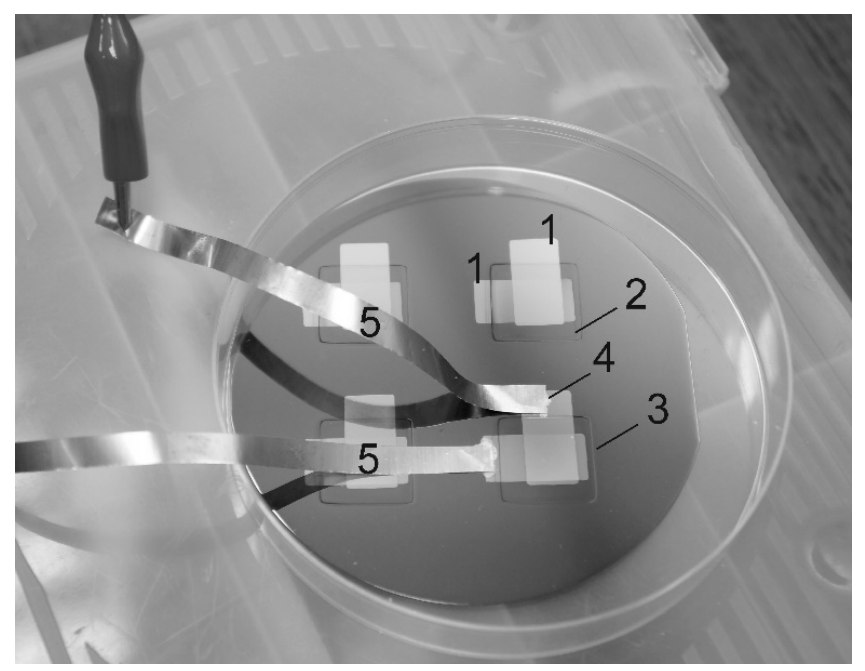

Figure 1. Experimental cells $\operatorname{Pt}(100 \mathrm{~nm}) / \mathrm{LiPON}(1 \mu \mathrm{m}) / \mathrm{Pt}(100 \mathrm{~nm})$ : 1—platinum electrodes; 2 -1 micron thick LiPON film; 3-silicon dioxide; 4-conductive glue; 5 -copper foil down conductors.

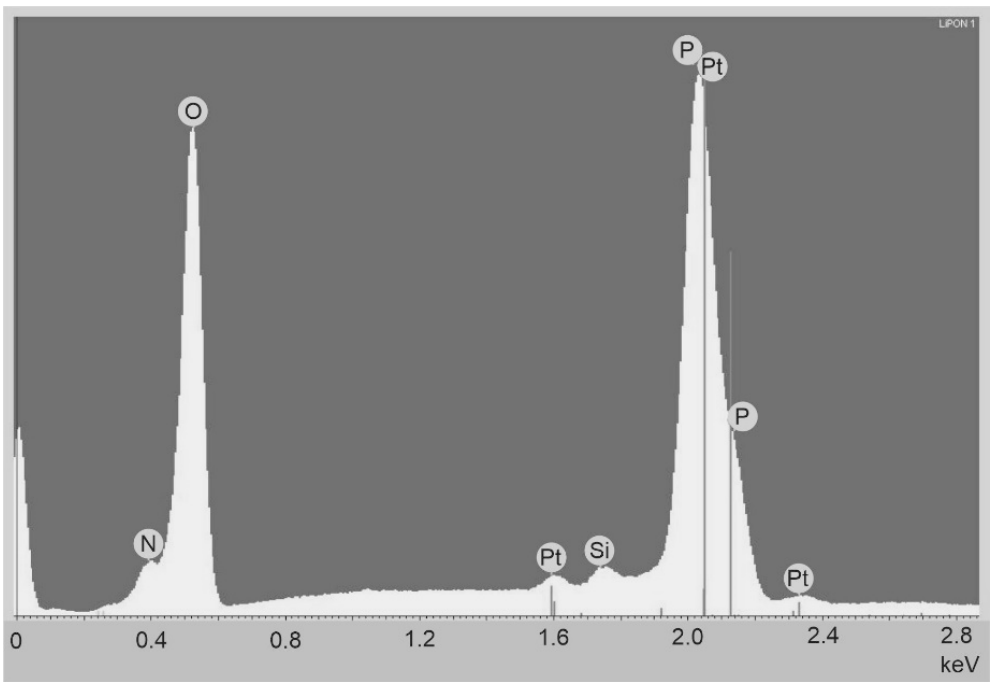

Figure 2. EDA (energy dispersive analysis) spectrum of solid LiPON electrolyte with platinum electrodes. Thin lines are platinum markers, where the marker height is proportional to the line intensity. The $\mathrm{Si}-\mathrm{K}_{\alpha}$ peak is due to the fluorescence of the silicon atom in the detector. 


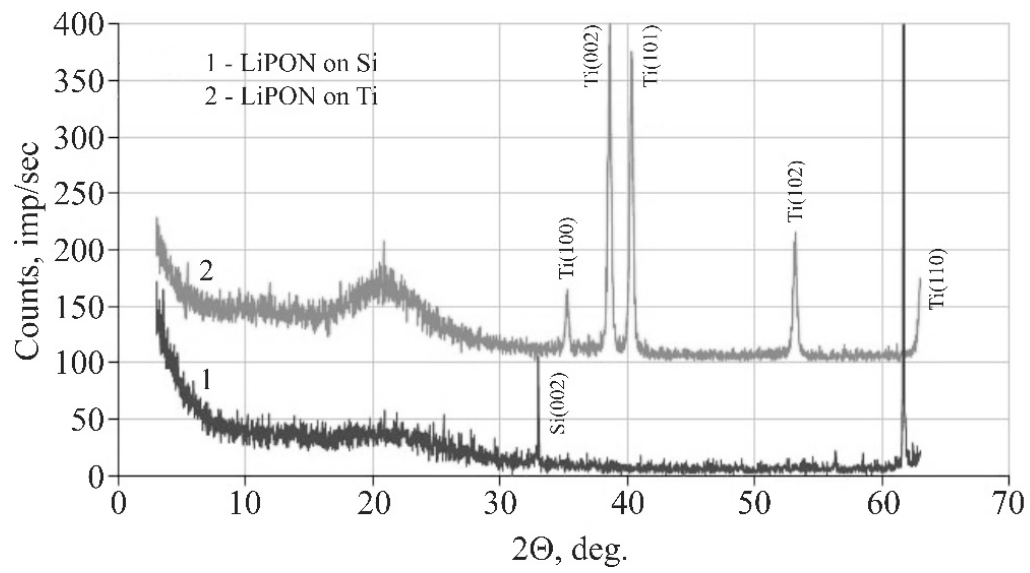

Figure 3. X-ray diffraction patterns of amorphous LiPON films on silicon wafer 1 and on titanium foil 2. The peaks in both curves belong to the substrates, while the amorphous LiPON gives a small halo in the region of peaks $2 \Theta=22.34^{\circ}$ and $2 \Theta=23.19^{\circ}$.

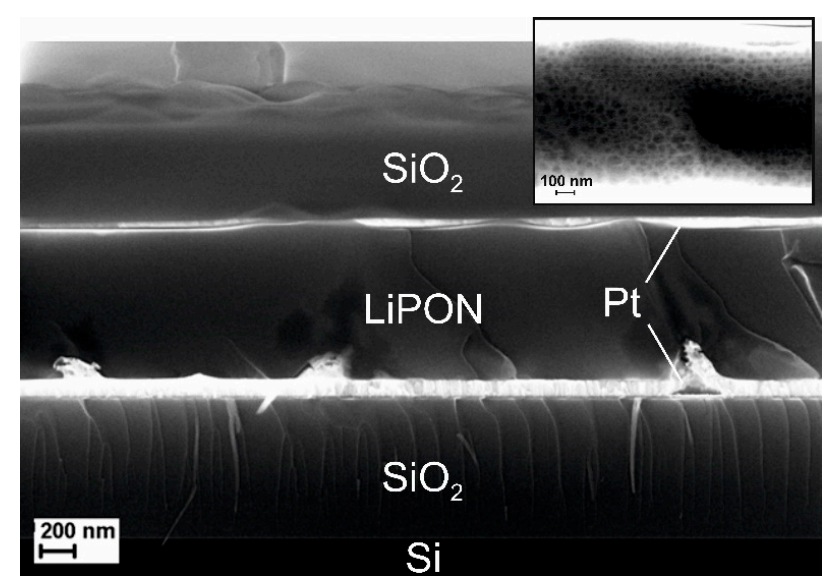

Figure 4. The cleavage of a $\mathrm{Pt}(100 \mathrm{~nm}) / \mathrm{LiPON}(1 \mu \mathrm{m}) / \mathrm{Pt}(100 \mathrm{~nm})$ cell (microscope Zeiss Supra 40). The inset shows a section of LiPON cleavage. The apparent porosity of LiPON in the inset is partly due to the microscopic products of lithium reaction, with atmospheric vapors and gases shown as dark spots.

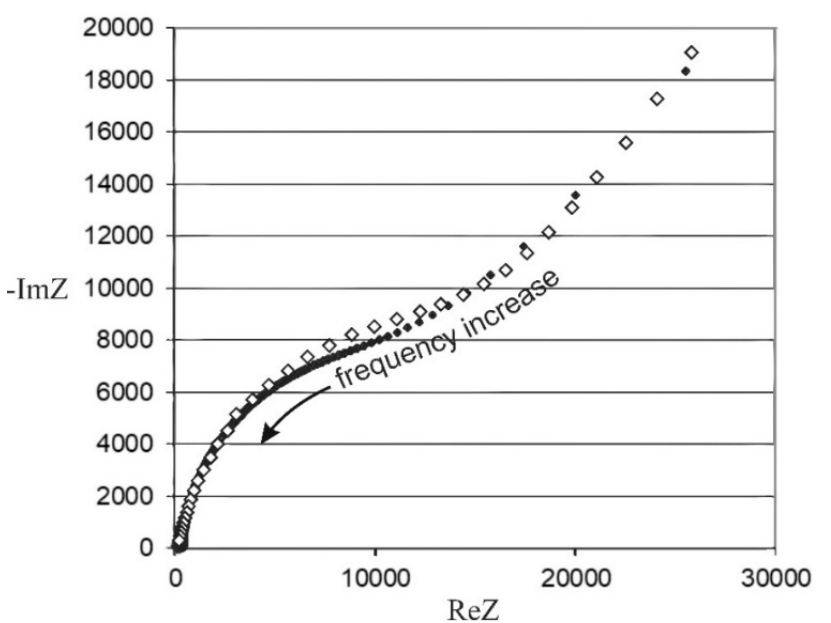

Figure 5. Experimental (open diamonds) and theoretical (black diamonds) Nyquist diagrams in the frequency range of $1 \mathrm{~Hz}$ to $20 \mathrm{kHz}$ for the parameters of the structural model $R_{e l}=180 \Omega$, $R_{l k}=11,000 \Omega, C_{d l}=2.1 \times 10^{-7} \mathrm{~F}, A_{W 1}=10^{4} \Omega \cdot \mathrm{s}^{-1 / 2}$, and $A_{W 2}=9 \times 10^{4} \Omega \cdot \mathrm{s}^{-1 / 2}$. 
To explain the features of the obtained spectrum, a structural model, shown in Figure 6, was proposed. When choosing a structural model, the following features of the $\mathrm{Pt} / \mathrm{LiPON} / \mathrm{Pt}$ cell were considered. First of all, it was taken into consideration that in the cell, the diffusion mechanism of charge transfer prevails. The lithium concentration gradient required to shield the external field was formed by lithium dissolved in platinum electrodes and the Faraday process. As was shown in [2], platinum electrodes contain a large amount of dissolved lithium and, depending on the polarity, extraction or insertion of lithium occurs. In addition, according to the results of [3], a Faraday process takes place on platinum electrodes, and the leakage current circuit is closed by the flow of the resulting lithium atoms. In other words, there are two oppositely directed diffusion flows: lithium ions and atoms.
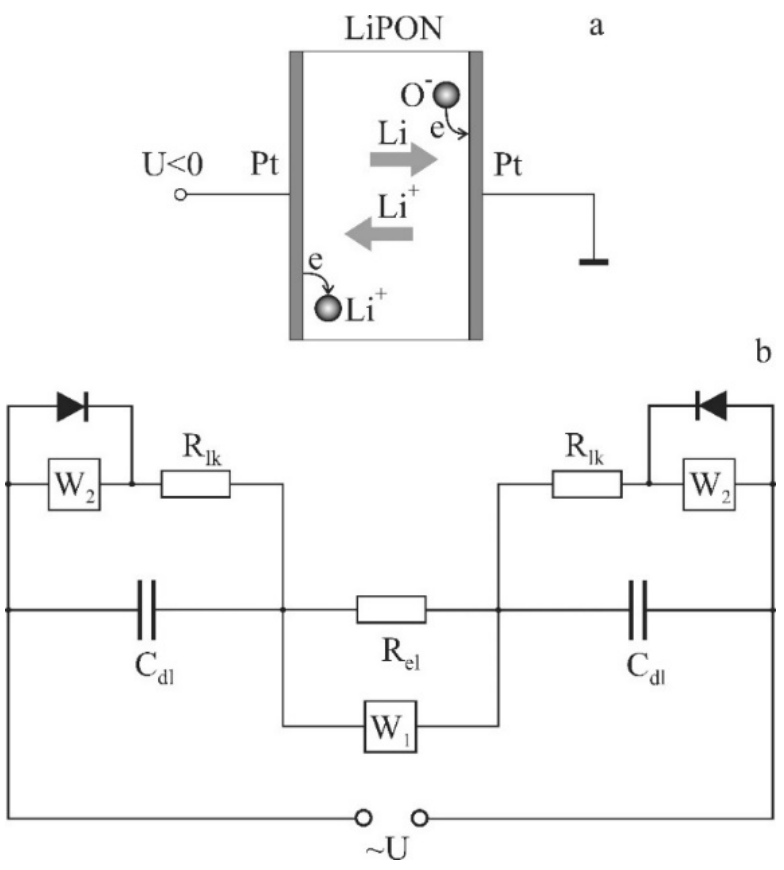

Figure 6. Structure of experimental cell $\mathrm{Pt} / \mathrm{LiPON} / \mathrm{Pt}$ : a-cell structure and direction of diffusion fluxes at constant voltage, and $\mathbf{b}$-structural model of LiPON.

The proposed structural model (Figure 6) consists of two capacitors corresponding to the capacitance of an electric double layer (EDL) on platinum electrodes, connected by an active resistance and a Warburg diffusion element. These elements simulate the drift and diffusion components of the polarization current. The Faraday process is simulated by a leakage current circuit connected in parallel to each of the capacitors and consists of an active resistance and a diffusion element. Since neutral lithium atoms are formed only at the cathode, diodes are connected in parallel to the diffusion elements, which shunt the Warburg diffusion element on the electrode, which, in this half-period is, the anode. Since it is not possible to calculate the impedance of a model with such structural elements as a diode, a simplified model is considered below.

In a simplified model (Figure 7), both EDLs were represented by one capacitor, $C_{d l} / 2$, and one leakage circuit. The current through the resistor, $R_{l k}$, simulates the redox reactions at the electrodes, while its value is determined by the rate of these reactions. The leakage current circuit is closed through the Warburg element, $W_{2}$, which simulates the resistance to the diffusion flux of lithium atoms moving from the cathode to the anode, where they are oxidized again. 


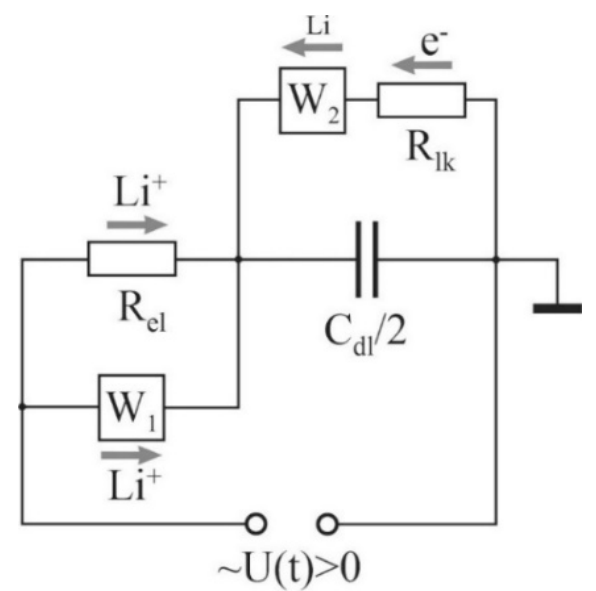

Figure 7. Simplified structural model of LiPON. The arrows show the direction of movement of charges at a positive potential on the electrode.

The impedance of the structural model shown in Figure 7, which is calculated in the same way as the resistance of parallel and series-connected conductors, has the form

$$
\begin{gathered}
\hat{Z}=\frac{A_{W 1}}{\sqrt{\omega}} R_{e l} \frac{(1-j)\left(R_{e l}+\frac{A_{W 1}}{\sqrt{\omega}}+j \frac{A_{W 1}}{\sqrt{\omega}}\right)}{\left(R_{e l}+\frac{A_{W 1}}{\sqrt{\omega}}\right)^{2}+\frac{A_{W 1}^{2}}{\omega}}- \\
-\frac{2}{\omega C_{d l}} \frac{\left[\frac{A_{W 2}}{\sqrt{\omega}}+j\left(R_{l k}+\frac{A_{W 2}}{\sqrt{\omega}}\right)\right]\left[R_{l k}+\frac{A_{W 2}}{\sqrt{\omega}}+j\left(\frac{A_{W 2}}{\sqrt{\omega}}+\frac{2}{\omega C_{d l}}\right)\right]}{\left(R_{l k}+\frac{A_{W 2}}{\sqrt{\omega}}\right)^{2}+\left(\frac{A_{W 2}}{\sqrt{\omega}}+\frac{2}{\omega C_{d l}}\right)^{2}}
\end{gathered}
$$

Separation of the real and imaginary parts of the impedance given by Equation (1) yields in the following expressions:

$$
\begin{aligned}
& \operatorname{Re} \hat{Z}=R_{e l} \frac{2+\frac{\sqrt{\omega} R_{e l}}{A_{W 1}}}{1+\left(1+\frac{\sqrt{\omega} R_{e l}}{A_{W 1}}\right)^{2}}+\left(\frac{2}{\omega C_{d l}}\right)^{2} \frac{R_{l k}+\frac{A_{W 2}}{\sqrt{\omega}}}{\left(R_{l k}+\frac{A_{W 2}}{\sqrt{\omega}}\right)^{2}+\left(\frac{A_{W 2}}{\sqrt{\omega}}+\frac{2}{\omega C_{d l}}\right)^{2}} \\
& \operatorname{Im} \hat{Z}=-j \frac{\frac{\sqrt{\omega} R_{e l}^{2}}{A_{W 1}}}{1+\left(1+\frac{\sqrt{\omega} R_{e l}}{A_{W 1}}\right)^{2}}-j \frac{2}{\omega C_{d l}} \frac{\left(R_{l k}+\frac{A_{W 2}}{\sqrt{\omega}}\right)^{2}+\frac{A_{W 2}}{\sqrt{\omega}}\left(\frac{A_{W 2}}{\sqrt{\omega}}+\frac{2}{\omega C_{d l}}\right)}{\left(R_{l k}+\frac{A_{W 2}}{\sqrt{\omega}}\right)^{2}+\left(\frac{A_{W 2}}{\sqrt{\omega}}+\frac{2}{\omega C_{d l}}\right)^{2}}
\end{aligned}
$$

where $A_{W}=U_{0} / \sqrt{2 D} S q c ; \Omega \cdot \mathrm{s}^{-1 / 2}$ is Warburg coefficient; $U_{0}, \mathrm{~V}$ is voltage amplitude across platinum electrodes; $D, \mathrm{~m}^{2} / \mathrm{s}$ is diffusivity coefficient; $S, \mathrm{~m}^{2}$ is electrode area; $q, \mathrm{C}$ is ion charge; $c, \mathrm{~m}^{-3}$ amplitude of oscillation of the nonequilibrium lithium concentration at the electrode surface.

\subsection{Diffusivity and Conductivity Calculation}

Equations (2) and (3) represent the parametric equation of a curve referred to as the Nyquist diagram. Figure 2, along with the experimental Nyquist diagram, shows a diagram calculated by Equations (2) and (3) for the following values of the parameters of structural elements: $R_{e l}=180 \Omega, R_{l k}=11,000 \Omega, C_{d l}=2.1 \times 10^{-7} \mathrm{~F}, A_{W 1}=10^{4} \Omega \cdot \mathrm{s}^{-1 / 2}$, and $A_{W 2}=9 \times 10^{4} \Omega \cdot \mathrm{s}^{-1 / 2}$. The value $R_{e l}=180 \Omega$ was obtained by the method described in [3] and was not used for fitting. The rest of the parameters were selected so that the deviation from the experimental curve, characterized by RSS (residual sum of squares), was minimal. Since the value of RSS itself does not characterize the quality of the approximation, it is expedient to use the normalized squared deviations, $\sigma^{2}(\operatorname{ReZ})=\left[\operatorname{Im} Z^{\exp }(\operatorname{ReZ})-\operatorname{Im} Z^{\mathrm{ap}}(\operatorname{ReZ})\right]^{2} /\left[\operatorname{Im} Z^{\exp }(\operatorname{Re} Z)\right]^{2}$, shown in Figure 8. 


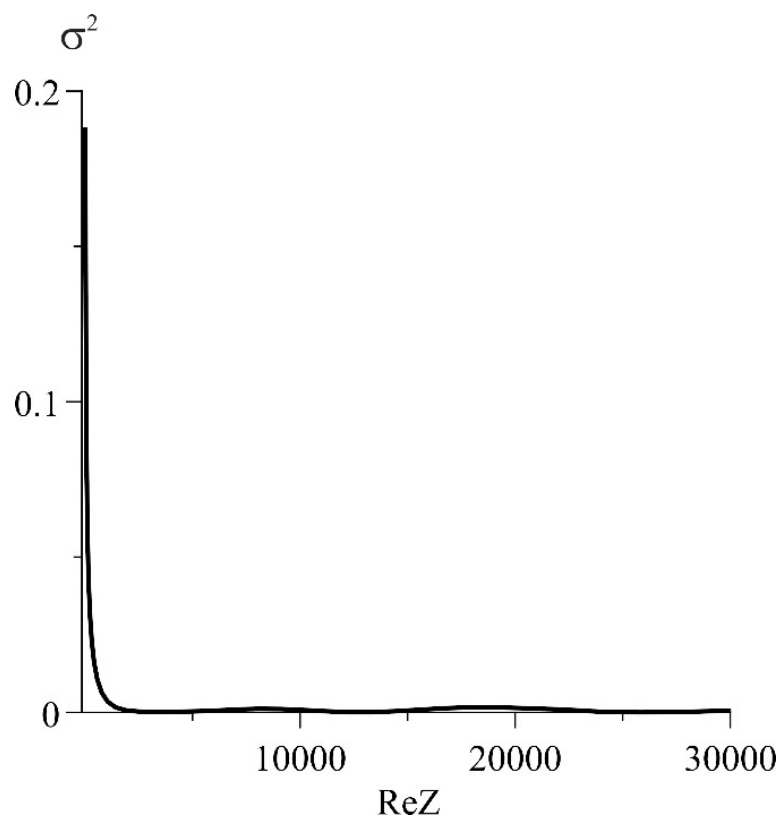

Figure 8. Dependence of the normalized squared deviations $\sigma^{2}$ on ReZ.

Note that, at high frequencies, the discrepancy between the experimental curve and its model increases. It is possible to achieve a more accurate coincidence of the experimental and calculated diagrams by introducing a constant phase element (CPE). This is the most commonly used approach. However, the structural models obtained in this case, in spite of the perfect coincidence of appropriate diagrams with the experimental curves, hardly lend themselves to physical interpretation. Therefore, in this work, the choice is made in the favor of the physical meaning, although with some damage to the accuracy of approximation of the experimental curves. For example, in the high-frequency region, according to Figure 7 , the capacitor $\left(C_{d l} / 2\right)$ shunts the elements $R_{l k}$ and $W_{2}$, and their variation does not affect the shape of the Nyquist diagram in any way. Therefore, it makes no sense to achieve full compliance of the model with the experimental curve in this area (Figure 8).

The value of the Warburg amplitude obtained by fitting was related to the diffusion coefficient by the equation

$$
A_{W 1}=\frac{U_{0}}{\sqrt{2 D_{1}} S q c_{0}}
$$

allowing us to find the diffusion coefficient of lithium ions, $D_{1}$. For this, it was necessary to know the amplitude of oscillation $\left(c_{0}\right)$ of their non-equilibrium concentration near the electrode. It is usually expressed from the Nernst equation for the electrode potential. In this case, $c_{0}$ can be expressed as the potential of a charged plane formed by an electrolyte layer with a thickness of $d_{\mathrm{Li}}$ located in the plane $x=0$ and placed in a medium of known dielectric permeability, $\varepsilon_{r}$. With respect to the grounded electrode located in the plane $x=\delta$, such a potential will be equal to $U_{0}=E \delta$, according to the Gauss theorem $E=d_{\mathrm{Li}} c_{0} q / 2 \varepsilon_{0} \varepsilon_{r}$. Then $c_{0}=2 \varepsilon_{0} \varepsilon_{r} U_{0} / d_{\mathrm{Li}} q \delta$, where $d_{\mathrm{Li}}$ is the diameter of the lithium ion, $q$ is the charge of the ion, and $\delta$ is the thickness of the LiPON layer. Expressing the diffusion coefficient from Equation (4) and substituting $c_{0}$, we obtain the final expression for the diffusion coefficient.

$$
D_{1}=\frac{1}{2}\left(\frac{d_{\mathrm{Li}} \delta}{A_{W 1} 2 S \varepsilon_{0} \varepsilon_{r}}\right)^{2}
$$

In [3], the relative dielectric permeability of LiPON was estimated as $\varepsilon_{r} \approx 250$, and the remaining parameters were $d_{\mathrm{Li}}=1.56 \times 10^{-10} \mathrm{~m}, \delta=10^{-6} \mathrm{~m}, A_{W 1}=10^{4} \Omega \cdot \mathrm{s}^{-1 / 2}$, and $S=6.4 \times 10^{-5} \mathrm{~m}^{2}$. Their substitution in Equation (5) gives the value of the diffusion coefficient $D_{1}=1.5 \times 10^{-11} \mathrm{~cm}^{2} / \mathrm{s}$, which is in good agreement with the data of $[4,5]$. 
The error in determining the diffusion coefficient can be estimated as follows. Logarithmic derivation of Equation (5) gave the dependence of the error in determining the diffusion coefficient on the Warburg amplitude of $\Delta D_{1} / D_{1} \approx 2 \Delta A_{1} / A_{1}$, since all other parameters were determined more accurately. In the course of fitting, the variation of the normalized $R S S=\sum_{i=1}^{N} \sigma_{i}^{2}$ in a vicinity of its minimum value, $R S S_{\min }=0.03345$, was $\approx 0.5 \%$, which corresponds to $2 \Delta A_{1} / A_{1} \approx 18 \%$. Thus, the final value of the diffusion coefficient will be as follows: $D_{1}=1.5( \pm 0.3) \times 10^{-11} \mathrm{~cm}^{2} / \mathrm{s}$.

The obtained value of the diffusion coefficient makes it possible to find the mobility of lithium ions, $\mu=5.8 \times 10^{-10} \mathrm{~cm}^{2} / \mathrm{V}$.s and calculate the conductivity of LiPON. The value of the concentration of lithium ions, $\mathrm{C}_{\mathrm{Li}^{+}}$, required for this calculation can be found on the basis of the leakage current, which is related to the concentration by the ratio

$$
\frac{C_{\mathrm{Li}^{+}} S d_{\mathrm{Li}} q}{\tau_{0}}=i_{l k}
$$

where $\tau_{0}$ is the relaxation time of the concentration of lithium ions due to reduction reactions at a cathode. In [3], the relaxation time during the discharge of $\mathrm{Pt} / \mathrm{LiPON} / \mathrm{Pt}$ through $1 \mathrm{M} \Omega$ was determined as $\tau_{0}=51.3 \mathrm{~s}$. In this case, Equation (6) gives the concentration value in the near-cathode layer by $C_{\mathrm{Li}^{+}}=2.1 \times 10^{22} \mathrm{~cm}^{-3}$. For the obtained values of the concentration and mobility of lithium ions, the conductivity was $\sigma=1.9 \times 10^{-6} \mathrm{~S} \cdot \mathrm{cm}^{-1}$. This value agrees with the data of [6], where the conductivity of LiPON was $2.3( \pm 0.7) \times 10^{-6} \mathrm{~s} \cdot \mathrm{cm}^{-1}$, as well as with the results of $[4,5]$ and a number of other works. It was noteworthy that the active resistance of LiPON, defined for a similar structure in [3] as $180 \Omega$, gives the conductivity value of $0.87 \times 10^{-6} \mathrm{~s} \cdot \mathrm{cm}^{-1}$.

The difference in the values of the amplitudes $A_{W 1}$ and $A_{W 2}$ was explained by the fact that $A_{W 1}$ simulates the complex resistance to the diffusion flux of lithium ions, and $A_{W 2}$ - to the diffusion flux of lithium atoms. Indeed, when the lithium ions reached the cathode, they were reduced to atoms, creating a concentration gradient and a diffusion flux directed from the cathode to the anode. Upon reaching the anode, the lithium atoms were oxidized, closing the leakage current circuit, and then moved in the opposite direction under the action of the electric field. The concentrations of lithium ions $\left(C_{\mathrm{Li}^{+}}=2.1 \times 10^{22}\right)$ and atoms $\left(C_{\mathrm{Li}} \sim 10^{19}\right)$ at the cathode [3] differ by three orders of magnitude. It can be expected that the amplitudes of the concentration oscillation differ by the same order, while the Warburg amplitudes differ by less than one order of magnitude. Hence, one can conclude that the diffusion coefficients of ions and atoms are also different.

\section{Conclusions}

A method for calculating the diffusion coefficient of lithium in solid electrolyte LiPON, based on the analysis of impedance spectroscopy data, was proposed. The essence of the method was to approximate the experimental Nyquist diagram by a curve obtained on the basis of the LiPON structural model and to determine the model parameters by the least squares method. One of the fitting parameters is the Warburg amplitude, which was related to the diffusion coefficient in Equation (5). This relationship was used to calculate the diffusion coefficient of lithium. The result obtained is in good agreement with the literature and data. This indicates the adequacy of the structural model of the solid electrolyte LiPON, which can be considered as an equivalent circuit.

Author Contributions: A.R.—writing of original draft, methodology; A.M.—methodology, investigation; V.N.-investigation, visualization; A.N.—investigation; A.S.—conceptualization, supervision; I.F.-investigation. All authors have read and agreed to the published version of the manuscript.

Funding: This research was funded by the Ministry of Science and Higher Education of the Russian Federation grant number 0856-2020-0006. 
Data Availability Statement: Supporting reported results data can be found or get by demand at The Facilities Sharing Centre "Diagnostics of Micro- and Nanostructures" (FSC DMNS), P.G. Demidov Yaroslavl State University.

Conflicts of Interest: The authors declare that they have no conflicts of interest. The funders had no role in the design of the study; in the collection, analyses, or interpretation of data; in the writing of the manuscript, or in the decision to publish the results.

\section{References}

1. Rudyi, A.S.; Mironenko, A.A.; Naumov, V.V.; Skundin, A.M.; Kulova, T.L.; Fedorov, I.S.; Vasil'ev, S.V. A Solid-State Lithium-Ion Battery: Structure, Technology, and Characteristics. Tech. Phys. Lett. 2020, 46, 217-221. [CrossRef]

2. Vasilev, S.V.; Lebedev, M.E.; Mazaletskii, L.A.; Metlitskayaa, A.V.; Mironenko, A.A.; Naumov, V.V.; Novozhilova, A.V.; Rudyi, A.S.; Fedorov, I.S. Development of the Technology of Magnetron Sputtering Deposition of LiPON Films and Investigation of Their Characteristics. Russ. Microelectron. 2017, 46, 424-432. [CrossRef]

3. Rudyi, A.S.; Lebedev, M.E.; Mironenko, A.A.; Mazaletskii LAm Naumov, V.V.; Novozhilova, A.V.; Fedorov, I.S.; Churilov, A.B. Study of the Relaxational Polarization Dynamics of the LiPON Solid Electrolyte. Russ. Microelectron. 2020, 49, 345-357. [CrossRef]

4. Put, B.; Vereecken, P.M.; Stesmans, A. On the chemistry and electrochemistry of LiPON breakdown. J. Mater. Chem. A 2018, 11, 4848-4859. [CrossRef]

5. Lacivita, V.; Artrith, N.; Ceder, G. Structural and compositional factors that control the li-ion conductivity in LiPON electrolytes. Chem. Mater. 2018, 30, 7077-7090. [CrossRef]

6. Yu, X.; Bates, J.; Jellison, G.; Hart, F. A stable thin-film lithium electrolyte: Lithium phosphorus oxynitride. J. Electrochem. Soc. 1997, 2, 524-532. [CrossRef] 